\title{
The minimal growth rate of cocompact Coxeter groups in hyperbolic 3-space
}

\author{
Ruth KELLERHALS* and Alexander KOLPAKOV**
}

\begin{abstract}
Due to work of W. Parry it is known that the growth rate of a hyperbolic Coxeter group acting cocompactly on $\mathbb{H}^{3}$ is a Salem number. This being the arithmetic situation, we prove that the simplex group $(3,5,3)$ has smallest growth rate among all cocompact hyperbolic Coxeter groups, and that it is as such unique. Our approach provides a different proof for the analog situation in $\mathbb{H}^{2}$ where E. Hironaka identified Lehmer's number as the minimal growth rate among all cocompact planar hyperbolic Coxeter groups and showed that it is (uniquely) achieved by the Coxeter triangle group $(3,7)$.
\end{abstract}

Keywords: Hyperbolic Coxeter group, growth rate, Salem number

\section{Introduction}

Let $G$ be a discrete group generated by finitely many reflections acting cocompactly on hyperbolic space $\mathbb{H}^{n}$. Denote by $S$ the set of generating reflections. We call $G=(G, S)$ an $n$-dimensional cocompact hyperbolic Coxeter group. A fundamental domain $P \subset \mathbb{H}^{n}$ for $G$ is a Coxeter polytope characterised by dihedral angles of the form $\pi / k$ for integers $k \geq 2$. In dimensions $n=2$ and $n=3$, there is a plethora of such hyperbolic Coxeter groups, which we can arrange by increasing volume of $P$. In this way, the right-angled triangle group $(3,7)$ is minimal and provides, by a result of Siegel $[\mathrm{Si}]$, an orbit space which is the over all smallest volume hyperbolic 2-orbifold. However, for $n=3$, it is known that the Coxeter tetrahedron with Coxeter symbol $(4,3,5)$ has smaller volume than its cousin $(3,5,3)$ (cf. $[\mathrm{Ke}]$ ), but the obvious internal 2-fold symmetry leads to an (arithmetic) discrete group $(3,5,3) \star \mathbb{Z}_{2}$ whose associated quotient has minimal volume (cf. [ChFr], [GM] and [MaM]). Another and more algebraic feature to characterise the set of hyperbolic Coxeter groups and to compensate the volume deficiency is the growth series with its growth rate. For a hyperbolic Coxeter group $G=(G, S)$, the growth series is given by

$$
f_{S}(x)=1+\sum_{k \geq 1} a_{k} x^{k}
$$

* Partially and **fully supported by Schweizerischer Nationalfonds 200020-131967.

2000 Mathematics Subject Classification. 20F55, 22E40, 51F15 
where $a_{k} \in \mathbb{Z}$ equals the number of words $w \in G$ with $S$-length $k$. The growth rate $\tau$ is defined by the reciprocal value of its radius of convergence $R \leq 1$. If $G$ is supposed to be cocompact, a result of Milnor [Mi] guarantees that $\tau=1 / R>1$. Furthermore, it is known that the series (1.1) is the power series of a rational function $p(x) / q(x)$ with coprime polynomials $p(x), q(x) \in \mathbb{Z}[x]$. By results of Floyd and Plotnick [FlPl] for $n=2$, and of Parry $[\mathrm{Pa}]$ for $n=2,3$, it follows that the denominator $q(x)$ is a product of cyclotomic polynomials with precisely one Salem polynomial (cf. $\$ 2.2$ ). Therefore, the growth rate $\tau$ is a Salem number, that is, a real algebraic integer $>1$ all of whose Galois conjugates have absolute value not greater than 1, and at least one has absolute value equal to one.

Lehmer's problem in number theory (see [GhHi]) can be formulated as minimisation problem and search for a uniform lower bound $s>1$ for all Salem numbers. By the above mentioned connection of Salem numbers to growth rates in dimensions 2 and 3, Lehmer's problem experiences a beautiful interpretation within hyperbolic geometry. For example, if $n=2$, E. Hironaka [Hi] proved that the group $(3,7)$ has minimal growth rate among all cocompact planar hyperbolic Coxeter groups, and that this Salem number $\tau_{1}$ equals Lehmer's number $\alpha_{L} \simeq 1.17628$ with minimal polynomial $L(x)=x^{10}+x^{9}-x^{7}-x^{6}-x^{5}-x^{4}-x^{3}+x+1$. Notice that $\alpha_{L}$ is the smallest Salem number known to date.

In this work, we prove that the tetrahedral Coxeter group $(3,5,3)$ has minimal growth rate among all of the infinitely many hyperbolic Coxeter groups acting cocompactly on $\mathbb{H}^{3}$. This Salem number $\tau_{2} \simeq 1.35098$ has minimal polynomial $x^{10}-x^{9}-x^{6}+x^{5}-x^{4}-$ $x+1$ (cf. (2.11)). The proof relies upon Steinberg's formula (cf. 2.5), a monotonicity property of the exponents of finite Coxeter groups (cf. Table 1) and some combinatorial and classification results for 3-dimensional cocompact Coxeter groups (cf. §2.1). The proof itself is nonetheless of elementary nature and requires, in contrast to $|S| \geq 6$, a limited case-by-case analysis for $|S|=4$ and $|S|=5$, only. It will be presented in full details revealing that the most delicate part arises when considering minimal $|S|$ and the growth rates of the rivaling Coxeter tetrahedra $(3,5,3)$ and $(4,3,5)$. Furthermore, the proof allows us to reprove Hironaka's result in the same spirit, without using any convexity argument. At the end, we shall illustrate that growth rates are not directly linked to covolume of hyperbolic Coxeter groups, but provide rather a good indicator for small covolume (cf. $\S 4)$.

Acknowledgement. This work was completed during the first author's research stay at the Osaka City University Advanced Mathematical Institute OCAMI (Osaka, Japan) and participation to the scientific program Geometric and Analytic Aspects of Group Theory at the Institut Mittag-Leffler (Djursholm, Sweden). She would like to express her warm thanks to the hosts. 


\section{Preliminaries}

2.1. Cocompact hyperbolic Coxeter groups. Let $\mathbb{X}^{n}$ be either the $n$-sphere $\mathbb{S}^{n}$, the Euclidean $n$-space $\mathbb{E}^{n}$ or the $n$-dimensional hyperbolic space $\mathbb{H}^{n}$. Let $P \subset \mathbb{X}^{n}$ be a Coxeter polytope, that is, $P$ is a convex polytope bounded by finitely many hyperplanes $H_{i} \subset \mathbb{X}^{n}, i \in I$, which - when intersecting in $\mathbb{X}^{n}$ - form dihedral angles $\angle\left(H_{i}, H_{j}\right)$ of type $\pi / k_{i j}$ for an integer $k_{i j} \geq 2$. Apart from the spherical case, hyperplanes bounding a polytope can be disjoint. In particular, hyperplanes bounding a finite volume polytope $P$ can be disjoint in $\mathbb{H}^{n}$ but intersect in the ideal boundary $\partial \mathbb{H}^{n}$ or admit a common perpendicular. In these cases, one sometimes speaks of (hyperbolically) parallel or ultraparallel hyperplanes.

The group $G=(G, S)=G(P)$ generated by the set $S$ of reflections $s_{i} \in \operatorname{Isom}\left(\mathbb{X}^{n}\right)$ with respect to the limiting hyperplanes $H_{i}$ of $P$ is called a geometric Coxeter group in $\mathbb{X}^{n}$. By construction, the generators $s_{i} \in S$ of $G$ provide the relations $s_{i}^{2}=1$ and $\left(s_{i} s_{j}\right)^{k_{i j}}=1$ if $\angle\left(H_{i}, H_{j}\right)=\pi / k_{i j}$ while $\left(s_{i} s_{j}\right)^{\infty}=1$ for (ultra-)parallel hyperplanes $H_{i}, H_{j}$. Furthermore, geometric Coxeter groups $G$ are discrete subgroups of $\operatorname{Isom}\left(\mathbb{X}^{n}\right)$ and provide an important class of geometric $n$-orbifolds $Q=\mathbb{X}^{n} / G$. We often do not distinguish between the geometric Coxeter group $G$ and its Coxeter polytope $P$ (or its quotient space $Q$ ). In the sequel, we always assume a hyperbolic Coxeter polytope to be compact and call the associated hyperbolic Coxeter group cocompact. A compact polytope $P \subset \mathbb{X}^{n}$ with non-obtuse dihedral angles is simple, that is, each $k$-dimensional face $F$ is contained in precisely $n-k$ bounding hyperplanes of $P$ for $0 \leq k \leq n-1$. In particular, if $P$ is spherical, then $P$ is a simplex, and the spherical Coxeter simplices have been classified by Coxeter $[\mathrm{Co}]$ in 1934 .

If a Coxeter group $(G, S)$ has a simple presentation, with few generators and relations, it is most convenient to represent $G$ by its Coxeter diagram $\Sigma$ or by its Coxeter symbol. Recall that the Coxeter diagram $\Sigma$ of $P$ (and $G$ ) consists of nodes corresponding to the hyperplanes of $P$ (or the reflections of $G$ ) which are pairwise connected by an edge of weight $k$ if the two corresponding hyperplanes intersect under the dihedral angle $\pi / k$. For the particular cases $k=2$ resp. $k=3$, the edge is always omitted resp. exempt from its weight. For a hyperbolic Coxeter polytope with ultra-parallel hyperplanes $H_{i}, H_{j}$ in its boundary (admitting a common perpendicular segment realising their distance $l_{i j}$ ), the corresponding nodes in $\Sigma$ are joined by a dotted edge (with weight $l_{i j}$ which is usually omitted). Furthermore, the Coxeter diagram of a hyperbolic Coxeter polytope of finite volume is connected. We call the number $|S|$ of generators the order of $\Sigma$.

The simplest geometric Coxeter groups in $\mathbb{X}^{n}$ are associated to linear Coxeter diagrams of order $n+1$ (and with weights $k_{1}, \ldots, k_{n}$, say). The corresponding polytope is called a Coxeter orthoscheme and denoted by the (slightly modified) Coxeter symbol $\left(k_{1}, \ldots, k_{n}\right)$. However, compact hyperbolic Coxeter orthoschemes exist only up to dimension 4, and the single compact examples in $\mathbb{H}^{3}$ are given by $(4,3,5),(3,5,3)$ and $(5,3,5)$, ordered by increasing volume (see $[\mathrm{Ke}]$, for example). In general, there are infinitely many, essentially 
different examples of compact Coxeter polyhedra in $\mathbb{H}^{2}$ and $\mathbb{H}^{3}$. This is obvious for $n=2$ since compact hyperbolic Coxeter $k$-gons $P \subset \mathbb{H}^{2}$ do exist as long as the total (interior) angle sum $\Omega$ of $P$ satisfies

$$
0<\Omega<(k-2) \pi .
$$

However, for $n=3$, this is much less obvious. By Andreev's theorem (see [Vi1, p. 112], for example), there are explicit realisation conditions which allow one to classify compact hyperbolic Coxeter polyhedra of fixed combinatorial type. For example, Coxeter simplices were classified by Lannér (see [Vi1, p. 205] for example), and the lists of triangular Coxeter prisms and hyperbolic Napier cycles are due to Kaplinskaja [Ka] and Im Hof [Im].

Example 1. The family

$$
\Sigma_{p, q, r}^{1} \quad: \quad \bullet \frac{p}{\bullet} \cdot \frac{q}{\bullet} \frac{r}{\bullet} \cdots \bullet \quad, \quad 1 / p+1 / q<1 / 2 \quad, \quad 1 / q+1 / r>1 / 2
$$

parametrised by three integers $p, q, r \geq 3$, yields an infinite sequence of compact truncated Coxeter orthoschemes (or orthogonal simplicial Coxeter prisms) in $\mathbb{H}^{3}$ discovered first by Kaplinskaja [Ka].

Example 2. The family of so-called Lambert cubes (see [Ke, p. 548 and p. 552] for the description and volume aspects) are particular doubly truncated orthoschemes and depend on three real parameters.

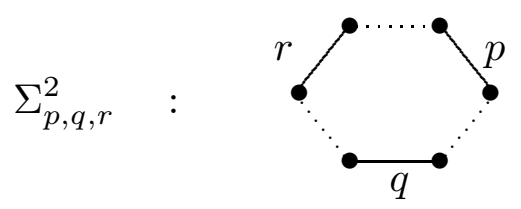

Figure 1.

They provide fundamental domains for cocompact reflection groups in $\operatorname{Isom}\left(\mathbb{H}^{3}\right)$ given by Coxeter graphs $\Sigma_{p, q, r}^{2}$ with integer weights $p, q, r \geq 3$ (see Figure 1) and were discovered by $\mathrm{Im}$ Hof $[\mathrm{Im}]$ in the context of Napier cycles.

2.2. Growth series and growth rates. Consider the growth series of a geometric Coxeter group $(G, S)$, that is,

$$
f(x)=f_{S}(x)=1+|S| x+\sum_{k \geq 2} a_{k} x^{k},
$$

where $a_{k}$ denotes the number of elements $w \in G$ of $S$-length $k$.

In the spherical case, growth of finite Coxeter groups is polynomial and well understood (see below). In the sequel, we are interested in the growth series of cocompact hyperbolic Coxeter groups $G$, which are of infinite order and may also have hyperbolic Coxeter subgroups (see Examples 1 and 2 above). Since $S=S^{-1}$, the coefficients satisfy $a_{k} \leq|S|^{k}$ so 
that the growth series (2.2) has radius of convergence $1 /|S| \leq R \leq 1$ in $\mathbb{C}$. Notice that, by a result of Milnor [Mi], $R<1$ since $G$ is cocompact. Furthermore, Steinberg [St] proved that $f(x)$ is a rational function of the form

$$
f(x)=\frac{p(x)}{q(x)} \quad, \quad \text { where } \quad p, q \in \mathbb{Z}[x]
$$

are monic and coprime polynomials of equal degree. In this way, we can interpret $f(x)$ as rational function of $x$ in the complex plane. The growth rate of $G$ is defined by $\tau=\tau_{G}:=$ $1 / R>1$ and coincides with the biggest positive real root of the denominator polynomial $q$ in (2.3). By definition, $\tau$ is a real algebraic integer. Our aim is to minimise $\tau$ for cocompact Coxeter groups in $\operatorname{Isom}\left(\mathbb{H}^{3}\right)$.

Consider a cocompact Coxeter group $G \subset \operatorname{Isom}\left(\mathbb{H}^{n}\right)$ for $n \geq 2$. In [CD, Corollary, p. 376], it is shown that the rational function $f(x)$ is reciprocal (resp. anti-reciprocal) for $n$ even (resp. $n$ odd), that is

$$
f\left(x^{-1}\right)=\left\{\begin{aligned}
f(x) & \text { for } \quad n \equiv 0(2) \\
-f(x) & \text { for } \quad n \equiv 1(2)
\end{aligned}\right.
$$

In particular, the inverse $\tau^{-1}=R<1$ is a Galois conjugate of the growth rate $\tau$. For its computation, the following formula of Steinberg [St] for arbitrary Coxeter groups $(G, S)$ will be of fundamental importance.

$$
\frac{1}{f_{S}\left(x^{-1}\right)}=\sum_{\substack{G_{T}<G \\\left|G_{T}\right|<\infty}} \frac{(-1)^{|T|}}{f_{T}(x)} .
$$

It provides an explicit procedure to compute $f_{S}\left(x^{-1}\right)$ in terms of the growth polynomials $f_{T}(x)$ of the finite Coxeter subgroups $G_{T}, T \subset S$, of $G$ where $G_{\varnothing}=\{1\}$. In this context, recall that any subset $T \subset S$ generates a Coxeter group $G_{T}$ which may be finite or infinite, reducible or irreducible. Combinatorially, each finite Coxeter subgroup $G_{T}<G$ arises as stabiliser of a certain face of $P$. In particular, a maximal finite Coxeter subgroup $G_{T}$ of $G$ acting on $\mathbb{H}^{n}$ is of rank $|T|=n$ and stabilises a vertex $v$ of $P$ whose link is a spherical $(n-1)$-simplex $P_{v}$ (by the simplicity of $P$ ). By a result of Solomon [So], the growth polynomial $f_{T}(x)$ is given by a product

$$
f_{T}(x)=\prod_{i=1}^{t}\left[m_{i}+1\right]
$$

Here we use the standard notations $[k]:=1+x+\cdots+x^{k-1},[k, l]=[k] \cdot[l]$ and so on. The entries $m_{1}=1, m_{2}, \ldots, m_{t}$ are the exponents of the Coxeter group $G_{T}$ (for references, see [CoMo, §9.7] or [D, Chapter 17], for example). In Table 1, we list the exponents and growth polynomials of the finite Coxeter groups of rank at most three. Finally, for a reducible 
finite Coxeter group $\left(G_{1} \times G_{2}, S_{1} \cup S_{2}\right)$, it is easily seen that its growth polynomial equals the product of the polynomials of $\left(G_{1}, S_{1}\right)$ and $\left(G_{2}, S_{2}\right)$. By $(2.5)$ and (2.6), it follows that the numerator polynomial $p(x)$ of $f(x)$ in $(2.3)$ is a product of cyclotomic polynomials. This is a consequence of the factorisation property (cf. [Pr, §3.3])

$$
[k]=\prod_{\substack{d \mid k \\ d>1}} \Phi_{d}(x)
$$

where $\Phi_{d}(x)$ denotes the $d$-th cyclotomic polynomial of degree equal to Euler's function $\varphi(d)$. For completeness, let us recall that the polynomial $\Phi_{d}(x)$ is irreducible in $\mathbb{Z}[x]$ and, for $d>2$, of even degree. If $p$ is prime and $d=p m$, it satisfies the property

$$
\Phi_{p m}(x)= \begin{cases}\Phi_{m}\left(x^{p}\right) & \text { if } p \mid m \\ \frac{\Phi_{m}\left(x^{p}\right)}{\Phi_{m}(x)} & \text { else }\end{cases}
$$

\begin{tabular}{|l|l|l|l|}
\hline Notation & Symbol & Exponents & $f_{S}(x)$ \\
\hline$A_{1}$ & - & 1 & {$[2]$} \\
$D_{2}^{k}, k \geq 2$ & $(k)$ & $1, k-1$ & {$[2, k]$} \\
$A_{3}$ & $(3,3)$ & $1,2,3$ & {$[2,3,4]$} \\
$B_{3}$ & $(4,3)$ & $1,3,5$ & {$[2,4,6]$} \\
$H_{3}$ & $(5,3)$ & $1,5,9$ & {$[2,6,10]$} \\
\hline
\end{tabular}

Table 1. Growth of irreducible finite Coxeter groups of rank at most 3

As another general fact, we mention that the growth series $f(x)$ of a Coxeter group $G$ acting cocompactly on $\mathbb{H}^{n}$ is related to the Euler characteristic of $G$ and the volume of $P$ as follows (see $[\mathrm{He}])$.

$$
\frac{1}{f(1)}=\chi(G)= \begin{cases}\frac{(-1)^{\frac{n}{2}} 2 \operatorname{vol}_{n}(P)}{\operatorname{vol}_{n}\left(\mathbb{S}^{n}\right)}, & \text { if } n \text { is even } \\ 0 & , \text { if } n \text { is odd } .\end{cases}
$$

Of special interest is the arithmetic nature and the distribution of the growth rates. By results of $[\mathrm{Ca}],[\mathrm{Pa}]$ (see also $[\mathrm{Hi}]$ ), it is known that the growth rate $\tau$ of a Coxeter group $G$ acting cocompactly on $\mathbb{H}^{n}$ is a Salem number if $n=2,3$. That is, $\tau$ is a real algebraic integer $>1$ all of whose conjugates have absolute value not greater than 1 , and at least one has absolute value equal to 1 . It follows that the minimal polynomial, called Salem polynomial, of $\tau$ is palindromic with $\operatorname{roots} \tau, \tau^{-1}$, and all other roots lie on the unit circle coming in inversive pairs. 
Example 3. Consider the famous triangle group

$$
\Gamma_{1}=(3,7) \quad: \quad \bullet-\bullet \frac{7}{-} \bullet
$$

with $|S|=3$ generating reflections acting cocompactly on $\mathbb{H}^{2}$. It is known that the group $\Gamma_{1} \subset \operatorname{Isom}\left(\mathbb{H}^{2}\right)$ is arithmetic and provides the (unique) hyperbolic 2-orbifold of minimal volume (cf. [Si]). Furthermore, $\Gamma_{1}$ is at the basis of the Hurwitz formula $\operatorname{Aut}\left(S_{g}\right) \leq$ $84(g-1)$ for the order of the automorphism group of a compact surface of genus $g>1$. The group $\Sigma_{1}$ has the rank 2 subgroups $D_{2}^{3}=A_{2}, D_{2}^{7}$ and $A_{1} \times A_{1}$ so that Steinberg's formula (2.5), combined with the reciprocity property (2.4), yields

$$
f_{(3,7)}(x)=\frac{[2,2,3,7]}{x^{10}+x^{9}-x^{7}-x^{6}-x^{5}-x^{4}-x^{3}+x+1} .
$$

By (2.7), the covolume of $\Sigma_{1}$ equals $\pi / 42$ as is also well known by the defect formula. The denominator of $f_{(3,7)}(x)$ is Lehmer's polynomial $L(x)$ which is the minimal polynomial of the smallest Salem number $\alpha_{L} \simeq 1.17628$ known to date. Hence, for the growth rate $\tau_{1}$ of $\Gamma_{1}$, we obtain $\tau_{1}=\alpha_{L}$.

Example 4. Consider the cocompact tetrahedral group

$$
\Gamma_{2}=(3,5,3) \quad: \quad \bullet-\bullet \frac{5}{-\bullet-\bullet}
$$

with $|S|=4$ generating reflections in $\operatorname{Isom}\left(\mathbb{H}^{3}\right)$. In order to apply Steinberg's formula (2.5), we list all finite subgroups with their growth polynomials according to Table 1 and summarise those of rank 2 and 3 in Table 2.

\begin{tabular}{|l|c|c|l|}
\hline Notation & Graph & Multiplicity & Polynomial \\
\hline$A_{1} \times A_{1}$ & $\bullet-\bullet$ & 3 & {$[2,2]$} \\
$A_{2}$ & $\bullet-\bullet$ & 2 & {$[2,3]$} \\
$D_{2}^{5}$ & $\bullet \frac{5}{5} \bullet$ & 1 & {$[2,5]$} \\
$A_{1} \times A_{2}$ & $\bullet-\bullet-\bullet$ & 2 & {$[2,2,3]$} \\
$H_{3}$ & $\bullet-\bullet-\bullet$ & 2 & {$[2,6,10]$} \\
\hline
\end{tabular}

Table 2. Finite subgroups of $(3,5,3)$

Then, by the anti-reciprocity (2.4), the growth function $f_{(3,5,3)}$ becomes (cf. also [W, p. 235])

$$
f_{(3,5,3)}(x)=\frac{[2,2,2,3] \cdot\left(x^{6}-2 x^{5}+3 x^{4}-3 x^{3}+3 x^{2}-2 x+1\right)}{(1-x) \cdot\left(x^{10}-x^{9}-x^{6}+x^{5}-x^{4}-x+1\right)}
$$


It follows that $x=1$ is a simple pole of $f_{(3,5,3)}(x)$ (cf. (2.7)), and the growth rate is the Salem number $\tau_{2} \simeq 1.35098>4 / 3$ associated to the minimal polynomial as given by the second factor of the denominator in (2.11). Let us add that in [W], the growth functions of all nine compact hyperbolic Coxeter simplex groups are listed and shown to have denominators which split into one Salem polynomial beside cyclotomic polynomials.

By exploiting Steinberg's formula for cocompact Coxeter groups $G \subset \operatorname{Isom}\left(\mathbb{H}^{n}\right)$ with fundamental polytope $P$, Floyd and Plotnick [FlPl] for $n=2$, and Parry [Pa] for $n=2,3$, succeeded to derive a closed formula for the growth function of $G$ in terms of the exponents associated to the (finite) vertex stabilisers. More precisely, the formula of Floyd and Plotnick for $n=2$ and a compact Coxeter polygon with interior angles $\pi / k_{1}, \ldots, \pi / k_{N}$ equals

$$
f(x)=\frac{[2] \cdot\left[k_{1}\right] \cdots\left[k_{N}\right]}{(x-N+1)\left[k_{1}\right] \cdots\left[k_{N}\right]+\sum_{i=1}^{N}\left[k_{1}\right] \cdots\left[\widehat{k_{i}}\right] \cdots\left[k_{N}\right]} .
$$

For $n=2$ and $n=3$, Parry provided an alternative characterisation, by considering $1 / f\left(x^{-1}\right)$ and by collecting the different contributions for each vertex $v$ of $P$ (with respective exponents $\left.m_{1}=1, m_{2}, m_{3}\right)$. For $n=3$, he obtained the beautiful expression

$$
\begin{aligned}
\frac{1}{f\left(x^{-1}\right)} & =: \frac{x-1}{x+1}+\sum_{v \in P} g_{v}(x) \\
& =\frac{x-1}{x+1}-\frac{1}{2} x(x-1) \sum_{v \in P} \frac{\left(x^{m_{1}}-1\right)\left(x^{m_{2}}-1\right)\left(x^{m_{3}}-1\right)}{\left(x^{m_{1}+1}-1\right)\left(x^{m_{2}+1}-1\right)\left(x^{m_{3}+1}-1\right)} .
\end{aligned}
$$

Based on the expressions (2.12) and (2.13), as well as on certain non-trivial properties about such rational functions, they could prove that the growth rate - in the cases $n=2,3$ - is always a Salem number.

\section{The main result}

Let $G$ be a Coxeter group acting cocompactly on hyperbolic space $\mathbb{H}^{n}$ and denote by $S$ its natural set of generating reflections. Let $P \subset \mathbb{H}^{n}$ be a fundamental Coxeter polytope for $G$. Since $G$ is cocompact, the polytope $P$ is simple. Let $\left(f_{0}, \ldots, f_{n-1}\right)$ denote its $f$-vector whose $k$-th component $f_{k}$ equals the number of $k$-dimensional faces of $P$. In particular, $f_{n-1}=|S|$. In the following, we consider the case $n=3$ and prove the following main result of this work.

Theorem. Among all Coxeter groups acting cocompactly on $\mathbb{H}^{3}$, the tetrahedral group $(3,5,3)$ has minimal growth rate, and as such the group is unique.

For the explicit shape of the growth function of $(3,5,3)$ giving rise to the Salem pole $\tau_{2} \simeq 1.35098$, recall from Example 4 and (2.11) that

$$
f_{(3,5,3)}(x)=\frac{[2,2,2,3] \cdot\left(x^{6}-2 x^{5}+3 x^{4}-3 x^{3}+3 x^{2}-2 x+1\right)}{(1-x) \cdot\left(x^{10}-x^{9}-x^{6}+x^{5}-x^{4}-x+1\right)} .
$$


3.1. Proof of the Theorem. Consider Parry's formula (2.13) for the growth function $f(x)$ of an arbitrary Coxeter group acting cocompactly on $\mathbb{H}^{3}$ which we rewrite by using (2.4) and $m_{1}=1$ so that

$$
\frac{1}{f(x)}=\frac{1-x}{1+x}\left\{1-\frac{x}{2} \sum_{v \in P} \frac{\left(x^{m_{2}}-1\right)\left(x^{m_{3}}-1\right)}{\left(x^{m_{2}+1}-1\right)\left(x^{m_{3}+1}-1\right)}\right\}
$$

and where the exponents $m_{2}, m_{3}$ are listed in Table 1. By having a closer look at Table 1, one can observe the following naive, but crucial fact. Let $G_{1} \neq A_{1}$ be a group in Table 1. By increasing one entry in the Coxeter symbol of $G_{1}$ and passing from $G_{1}$ to another group $G_{2}$ in Table 1, the non-trivial exponents all increase as well. For example, the passage from $A_{3}$ to $H_{3}$ (increase the first entry 3 of the Coxeter symbol $(3,3)$ to 5 ) transforms the exponents different from $m_{1}=1$ according to $m_{2}: 2 \mapsto 5$ and $m_{3}: 3 \mapsto 9$. In order to make use of this observation, we start by modifying (3.1) and write

$$
\frac{1}{f(x)}=\frac{1-x}{[2]}\left\{1-\frac{x}{2} \sum_{v \in P} h_{k}(x) h_{l}(x)\right\}=: \frac{1-x}{[2]}\left\{1-\frac{x}{2} H(x)\right\}
$$

interpreting the exponents $m_{2}$ and $m_{3}$ associated to the stabiliser of each vertex $v \in P$ as certain positive integers denoted simply by $k$ and $l$, and where

$$
h_{k}(x)=h_{k}^{v}(x)=\frac{x^{k}-1}{x^{k+1}-1}=\frac{[k]}{[k+1]} \text {, }
$$

which is strictly monotonely decreasing on $[0,1]$ and satisfies the following simple but important property.

Lemma. For each integer $k \geq 1$ and for all $x \in(0,1], \quad 0<h_{k}(x)<h_{k+1}(x)<1$.

Proof. We have

$$
\frac{1}{h_{k}(x)}-\frac{1}{h_{k+1}(x)}=x^{k}\left(\frac{1}{[k]}-\frac{x}{[k+1]}\right),
$$

whose positivity on $(0,1]$ is equivalent to the trivial inequality $1+x+\cdots+x^{k}>x+x^{2}+$ $\cdots+x^{k}$.

Our strategy is to show that, for each $x \in\left(0,1 / \tau_{2}\right]$, there is a strict inequality $1 / f(x)<$ $1 / f_{(3,5,3)}(x)$ for the growth function $f$ of any three-dimensional cocompact hyperbolic Coxeter group different from $(3,5,3)$. Equivalently, by (2.11) and (3.2), we will show that

$$
H(x)>H_{(3,5,3)}(x)=2\left\{\frac{1}{[3]}+\frac{[5,9]}{[6,10]}\right\} \quad \text { for } \quad x \in\left(0,1 / \tau_{2}\right] .
$$

Consider a compact Coxeter polyhedron $P \subset \mathbb{H}^{3}$ associated to $G$. Obviously, $P$ must have $f_{2} \geq 4$. Furthermore, we know that $P$ is simple, that is, $3 f_{0}=2 f_{1}$. Euler's polyhedron 
formula $f_{0}-f_{1}+f_{2}=2$ then yields the identity $f_{0}=2\left(f_{2}-2\right)$ (or $\left.f_{1}=3\left(f_{2}-2\right)\right)$ in terms of $f_{2}=|S|$. We shall prove (3.4) by distinguishing between the three cases $f_{2}=4$, $f_{2}=5$ and $f_{2} \geq 6$.

Suppose that $f_{2} \geq 6$. If $P$ is totally right-angled, then, by a result of Inoue [In, Lemma 3.3], $f_{2} \geq 12$, and therefore $f_{0} \geq 20$. Since $m_{2}=m_{3}=1$, we get the estimate

$$
H(x) \geq \frac{20}{[2]^{2}}=: \widetilde{H}(x)
$$

We will prove the inequality $\widetilde{H}(x)>H_{(3,5,3)}(x)$, which is stronger than (3.4), by making the following observation. For any positive integer $l$,

$$
\frac{2}{[2]}>\frac{[l]}{[l+1]} \quad \text { for } \quad x \in[0,1]
$$

since (3.6) is equivalent to the inequality $x^{l}+1>0$. Now, by (3.6), we get

$$
\widetilde{H}(x)=\frac{20}{[2]^{2}}>\frac{4}{[2]^{2}}+2\left\{\frac{[2]}{[2,3]}+\frac{[5,9]}{[6,10]}\right\}>2\left\{\frac{[2]}{[2,3]}+\frac{[5,9]}{[6,10]}\right\}=H_{(3,5,3)}(x)
$$

Suppose that $P$ has at least one dihedral angle $\pi / l$ with an integer $l \geq 3$, associated to the edge $e=F \cap F^{\prime}$ formed by two faces $F, F^{\prime}$ bounding $P$, say. Let $v, v^{\prime}$ be the vertices on the edge $e$ of $P$. By looking at their vertex figures, it follows that both stabiliser subgroups of $v$ and $v^{\prime}$ are finite Coxeter groups of the form $(k, l)$ and $\left(k^{\prime}, l\right)$ with certain integers $k, k^{\prime} \geq 2$ (see also Table 1 ). By the Lemma, and since $f_{0} \geq 8$, this leads to the rough estimate

$$
H(x) \geq \frac{6}{[2]^{2}}+2 \frac{[l-1]}{[2, l]} \geq \frac{6}{[2]^{2}}+\frac{2}{[3]}=: \widetilde{H}(x) \quad .
$$

In order to show that $\widetilde{H}(x)>H_{(3,5,3)}(x)$, we need to verify that (see (3.4) and $\S 2.2$ )

$$
\frac{3}{[2]^{2}}>\frac{[5,9]}{[6,10]}=\frac{x^{6}+x^{3}+1}{x^{8}+x^{5}+x^{3}+1}
$$

which is equivalent to the evident positivity (on $[0,1$ ), say) of the difference polynomial

$$
\begin{aligned}
\Delta(x) & =3\left(x^{8}+x^{5}+x^{3}+1\right)-(x+1)^{2}\left(x^{6}+x^{3}+1\right) \\
& =(1-x)^{2}(1+x)^{2} \cdot\left(2 x^{4}-2 x^{3}+3 x^{2}-2 x+2\right) \\
& =(1-x)^{2}(1+x)^{2} \cdot\left(2(1-x)\left(1+x^{2}\right)+x^{2}\left(1+2 x^{2}\right)\right)>0 .
\end{aligned}
$$

Suppose that $f_{2}=5$. Then, $P$ is a Coxeter prism which is combinatorially the product of a 1 -simplex with a 2 -simplex and which has $f_{0}=6$ vertices. The associated Coxeter groups $G$ are given by a Coxeter diagram which contains exactly one dotted edge (corresponding to 
an infinite subgroup). Each Coxeter prism arises by glueing straight Coxeter prisms, which are distinguished by one (top) facet which is totally orthogonal to all its incident (lateral) facets. Based on this observation, Kaplinskaja [Ka] classified all straight Coxeter prisms (for a complete list, cf. [Vi2, Table 4, p. 61]). In Table 3, we summarise the relevant existence data for straight Coxeter prisms $G=: P_{p, q, r, t}$ in terms of integer parameters $p, q, r, t$ (cf. also $§ 2.1$, Example 1) as well as for the two additional examples $G=: Q_{4}, Q_{5}$. Observe that the cyclic subdiagram, formed by the edges with weights $p, q, r$ in $P_{p, q, r, t}$, is hyperbolic while the subdiagrams with Coxeter symbols $(t, p)$ and $(t, r)$ are spherical.

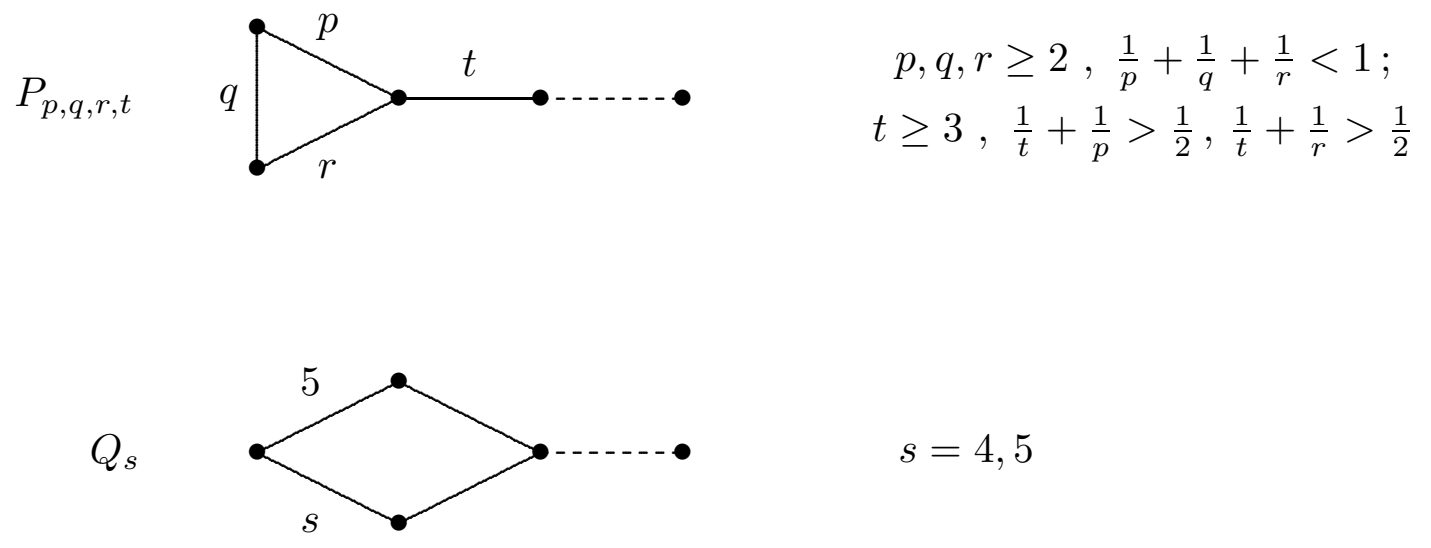

Table 3. The compact straight Coxeter prisms in $\mathbb{H}^{3}$

Notice that the Lemma allows us - as in (3.7) - to restrict the proof of the Theorem for $f_{0}=5$ to straight Coxeter prisms $P_{\sigma}$. More precisely, for the auxiliary function $\tilde{H}(x):=H_{P_{\sigma}}(x)$, we have $H_{G}=H(x) \geq \tilde{H}(x)$ on $(0,1]$ by the Lemma, and we shall prove that $\tilde{H}(x)>H_{(3,5,3)}(x)$ on $\left(0,1 / \tau_{2}\right]$.

We start with the $Q$-examples. Again by the Lemma, it suffices to treat only the case $Q_{4}$. The Lemma and (3.8) yield

$$
\begin{aligned}
H(x) & =\frac{[2,3]}{[3,4]}+\frac{[3,5]}{[4,6]}+\frac{[5,9]}{[6,10]}+\frac{1}{[2]^{2}}+\frac{[3]}{[2,4]}+\frac{[4]}{[2,5]} \\
& >\frac{2}{[2]^{2}}+\frac{[5,9]}{[6,10]}+\frac{1}{[2]^{2}}+\frac{2}{[3]}>2\left\{\frac{1}{[3]}+\frac{[5,9]}{[6,10]}\right\},
\end{aligned}
$$

which proves the desired inequality $H(x)>H_{(3,5,3)}(x)$.

For the $P$-series, we consider the two cases $q=2$ and $q \geq 3$. For $q=2$, the conditions in Table 3 imply that $P$ is of the form $P=P_{p, 2,5,3}$ with $p=4,5$. By the Lemma, we investigate the case $p=4$, only, and can bound $H(x)$ as follows.

$$
\begin{aligned}
H(x) & =\frac{[3,5]}{[4,6]}+\frac{[5,9]}{[6,10]}+\frac{2}{[2]^{2}}+\frac{[3]}{[2,4]}+\frac{[4]}{[2,5]} \\
& >\frac{1}{[3]}+\frac{[5,9]}{[6,10]}+\frac{2}{[2]^{2}}+\frac{1}{[3]}+\frac{1}{[2]^{2}}=\frac{2}{[3]}+\frac{[5,9]}{[6,10]}+\frac{3}{[2]^{2}} .
\end{aligned}
$$


By (3.8), the inequality (3.10) yields (3.4), as desired.

Consider now the case $q \geq 3$. By the Lemma, it suffices to consider $p=2$ and linear Coxeter diagrams of type (see $\S 2.1$, Example 1 )

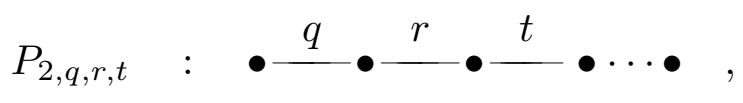

where the parameters $q, r, t \geq 3$ satisfy the additional conditions of Table 3 . Together with the Lemma, these conditions allow us to suppose that $t=3$ (see also [Ka, p. 89]). Then, either $r=3, q \geq 7$ or $r \geq 4, q \geq 4$ (except $r=q=4$ ). In both cases, the hyperbolicity of the subgraph $(q, r)$ and the Lemma yield the estimate

$$
H(x) \geq \frac{[m(r), m(3)]}{[m(r)+1, m(3)+1]}+\frac{1}{[2]^{2}}+2 \frac{[q-1]}{[2, q]}+\frac{[r-1]}{[2, r]}+\frac{1}{[3]},
$$

where $m(r), m(3)$ denote the exponents of the finite Coxeter group $(r, 3)$ (cf. Table 1). Consider the first case with $r=3$ and $q \geq 7$. By applying (3.6) to the third term in (3.11), and by the Lemma, we obtain

$$
H(x) \geq \frac{[2,3]}{[3,4]}+\frac{1}{[2]^{2}}+\frac{[6,9]}{[7,10]}+\frac{2}{[3]}>\frac{[2]}{[4]}+\frac{1}{[2]^{2}}+\frac{[5,9]}{[6,10]}+\frac{2}{[3]}
$$

But the inequality

$$
\frac{[2]}{[4]}+\frac{1}{[2]^{2}}>\frac{[5,9]}{[6,10]}
$$

is equivalent to the evident inequality of the difference polynomial

$$
\Delta(x)=(1-x)^{2}[2]^{3}[3,5] \cdot\left(1+x^{6}+x^{2} \cdot\left(2\left\{1+x^{2}\right\}-x\right)>0 \quad \text { on } \quad[0,1)>0 \quad .\right.
$$

Therefore, $H(x)>H_{(3,5,3)}(x)$ as desired.

In the second case with $r, q \geq 4$, we may assume that $q=4$ and $r=5$ when looking at the middle term in (3.11). Then, by the Lemma and (3.8) allow us to transform (3.11) as follows.

$$
H(x) \geq \frac{[5,9]}{[6,10]}+\frac{1}{[2]^{2}}+2 \frac{[3]}{[2,4]}+\frac{[4]}{[2,5]}+\frac{1}{[3]}>\frac{[5,9]}{[6,10]}+\frac{3}{[2]^{2}}+\frac{2}{[3]}>2 \frac{[5,9]}{[6,10]}+\frac{2}{[3]}
$$

which proves (3.4) for $f_{2}=5$.

Suppose that $f_{2}=4$. In this case, $P$ is a Coxeter tetrahedron and $G$ is one of the nine Lannér groups as given in Table 4 (cf. [Vi, p. 205] and [W] for their growth functions). We will show as above that, for each Coxeter group $G \neq R_{1}=(3,5,3)$ in Table 4 , we have $H(x)>H_{(3,5,3)}(x)$ for $x \in\left(0,1 / \tau_{2}\right]$. By the Lemma, it suffices to do this for the $S$-series, for $T$ and the $R$-series separately, and for the Coxeter diagrams with smallest weights in each series, only. 

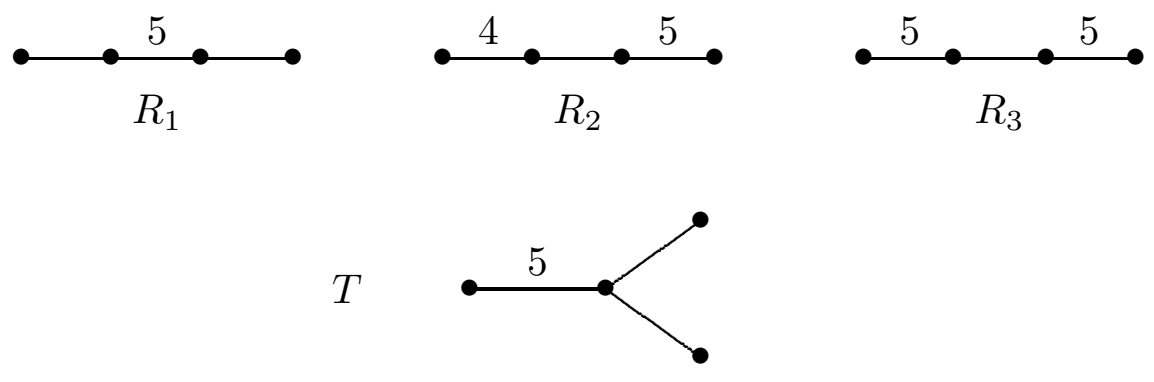

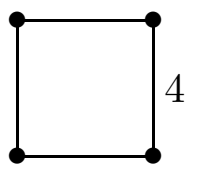

$S_{1}$

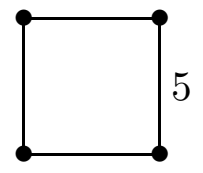

$S_{2}$

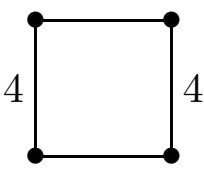

$S_{3}$

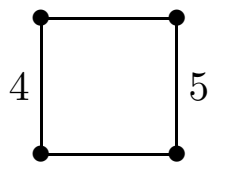

$S_{4}$

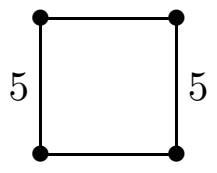

$S_{5}$

Table 4. The Lannér groups

Let us start with the $S$-series and consider $S_{1}$ which has the smallest weights in this set. More specifically, $S_{1}$ has among its four finite subgroups of rank three the group $A_{3}$ with multiplicity two and the group $B_{3}$ with multiplicity two. By $(2.5)$ and by Table 1 , we can compute $H(x)$ and, by using the Lemma and (3.6), we bound it as follows.

$$
H(x)=2 \frac{[2,3]}{[3,4]}+2 \frac{[3,5]}{[4,6]}>2 \frac{[2]}{[2,3]}+2 \frac{[5]}{[2,6]}>\frac{2}{[3]}+2 \frac{[5,9]}{[6,10]}=H_{(3,5,3)}(x)
$$

for each $x \in(0,1]$.

Consider next the diagram $T$. In a similar way, we get

$$
H(x)=2 \frac{[5,9]}{[6,10]}+\frac{[2,3]}{[3,4]}+\frac{1}{[2]^{2}}
$$

Since $[4]=[2]\left(x^{2}+1\right)$ by $\S 2.2$, and by the Lemma, we can treat the last two terms according to

$$
\frac{[2]^{2}+x^{2}+1}{[2]^{2}\left(x^{2}+1\right)}=2 \frac{[3]}{[2]^{2}\left(x^{2}+1\right)}=2 \frac{[3]}{[2,4]}>\frac{2}{[3]},
$$

which yields the desired inequality (3.4).

The $R$-series and especially $R_{2}=(4,3,5)$ are the most delicate cases. For $R_{2}$, we compute

$$
H(x)=\frac{[3,5]}{[4,6]}+\frac{[5,9]}{[6,10]}+\frac{[3]}{[2,4]}+\frac{[4]}{[2,5]} .
$$

We have to show that, for $x \in\left(0,1 / \tau_{2}\right] \subset(0,3 / 4]$,

$$
\frac{[3,5]}{[4,6]}+\frac{[3]}{[2,4]}+\frac{[4]}{[2,5]}>\frac{2}{[3]}+\frac{[5,9]}{[6,10]}
$$


which we do by using the divisibility properties of $[k]$ (cf. $§ 2.2$ ) and by considering the difference $\Delta(x)$ of the two rational functions in (3.12). In this way, an easy computation yields

$$
\Delta(x)=\frac{2 x^{2}\left(x^{8}-x^{5}-x^{4}-x^{3}+1\right)}{[2,2,3,5]\left(x^{2}+1\right) \cdot\left(x^{4}-x^{3}+x^{2}-x+1\right)}=: \frac{2 x^{2} p(x)}{q(x)} .
$$

For the factor $r(x)=x^{4}-x^{3}+x^{2}-x+1$ of $q(x)$ in (3.13), we write $r(x)=x^{4}+$ $x^{2}+1-x\left(x^{2}+1\right)=x^{4}+(1-x)\left(x^{2}+1\right)$ which is clearly positive on $[0,1)$. For the numerator polynomial $p(x)$ in (3.13), however, observe that $p(1)<0$. We write $p(x)=$ $x^{8}+1-x^{3}\left(x^{2}+x+1\right)=: a(x)-b(x)$ and consider the polynomials $a(x), b(x)$ on the interval $[0,3 / 4]$. For $0 \leq x \leq 3 / 4$, we have $a(x)>1$, while

$$
b(x)=x^{3}\left(x^{2}+x+1\right) \leq b(3 / 4)=\frac{999}{4^{5}}<\frac{10^{3}}{4^{5}}=\frac{125}{128}<1,
$$

that is, $p(x)>0$. Hence, it follows that $\Delta(x)>0$ on $(0,3 / 4]$ which finishes the proof.

3.2. An analog proof in dimension two. By the same method, the result of E. Hironaka $[\mathrm{Hi}]$ in two dimensions can be derived in a more elementary way, and without using a convexity argument for the function (2.12). More precisely, consider a compact Coxeter polygon $P \subset \mathbb{H}^{2}$ with $f_{0}=|S|$ vertices and its associated Coxeter group $G$. In order to prove that the minimal growth rate $\tau_{1}$ is (uniquely) attained by the triangle group $\Gamma_{1}=(3,7)$ (see Example 3), and hence $\tau_{1}=\alpha_{L} \simeq 1.17628$, we consider Steinberg's expression $(2.5)$

$$
\frac{1}{f\left(x^{-1}\right)}=\sum_{\substack{G_{T}<G \\\left|G_{T}\right|<\infty}} \frac{(-1)^{|T|}}{f_{T}(x)} .
$$

By reciprocity (2.4), $f\left(x^{-1}\right)=f(x)$. As in $\S 3.1$, we look at the $f_{0}$ vertices $v \in P$. Denote by $\pi / k_{v}$ the interior angle at $v$ in $P$. That is, the vertex stabiliser $G_{v} \subset G$ is the dihedral group $D_{2}^{k_{v}}$ of order $2 k_{v} \geq 4$, with Coxeter symbol $\left(k_{v}\right)$ and growth polynomial $\left[2, k_{v}\right]$. Furthermore, since $|S|=f_{0}$, the equation (3.14) can be simplified according to

$$
\begin{aligned}
\frac{1}{f(x)} & =1-\frac{|S|}{[2]}+\sum_{v \in P} \frac{1}{\left[2, k_{v}\right]}=1-\frac{1}{[2]} \sum_{v \in P}\left\{1-\frac{1}{\left[k_{v}\right]}\right\} \\
& =1-\frac{x}{[2]} \sum_{v \in P} \frac{\left[k_{v}-1\right]}{\left[k_{v}\right]}=: 1-\frac{x}{[2]} \sum_{v \in P} h_{v}(x),
\end{aligned}
$$

where the auxiliary functions $h_{v}, v \in P$, and their sum can be written in the form (cf. $(3.3))$

$$
h_{k}(x)=h_{k_{v}}(x)=\frac{[m(k)]}{[m(k)+1]} \quad, \quad H(x):=\sum_{v \in P} h_{k}(x),
$$


since the exponents of the group $D_{2}^{k_{v}}$ are equal to $m_{1}=1$ and $m_{2}=m(k)=k_{v}-1$. By the Lemma, we have that $0<h_{i}(x)<h_{j}(x)<1$ for all $i<j$ and for all $x \in(0,1]$.

We want to show that for each $x \in\left(0,1 / \alpha_{L}\right]$, the value $1 / f_{(3,7)}(x)$ is strictly bigger than the corresponding value $1 / f(x)$ for any compact Coxeter polygon $P \subset \mathbb{H}^{2}$ which is not isometric to the triangle $(3,7)$. By $(3.15)$ and $(3.16)$, this means that we have to show that

$$
H(x)>H_{(3,7)}(x)=\frac{1}{[2]}+\frac{[2]}{[3]}+\frac{[6]}{[7]} \quad \text { for all } \quad x \in\left(0,1 / \alpha_{L}\right] \quad .
$$

First, we consider the case of compact Coxeter triangles $P$ with angular existence condition $1 / p+1 / q+1 / r<1$ for integers $p, q, r \geq 2$ (cf. (2.1)). Hence, at most one angle of $P$ can be equal to $\pi / 2$. Consider The angular existence condition and the Lemma imply that $H$ must satisfy at least one of the following inequalities.

$$
\begin{aligned}
& H(x) \geq \frac{1}{[2]}+\frac{[2]}{[3]}+\frac{[l]}{[l+1]}=: H_{l}(x) \quad \text { for } \quad l \geq 6, \\
& H(x) \geq \frac{1}{[2]}+\frac{[3]}{[4]}+\frac{[4]}{[5]}=: H_{4}(x)>H_{(3,7)}(x),
\end{aligned}
$$

with equality in (3.18a) only if $l=6$ and therefore $G \cong \Gamma_{1}$. Indeed, the first inequality holds for all Coxeter triangles having angles $\pi / 2, \pi / 3$, and by comparison with (3.17), the function $H_{l}(x), l \geq 6$, does coincide with $H_{(3,7)}(x)$ precisely for $l=6$. As for (3.18b), which concerns right-angled Coxeter triangles with no angle equal to $\pi / 3$, we consider the difference function $\Delta(x):=H_{4}(x)-H_{(3,7)}(x)$ for $x \in(0,1]$. A straightforward computation yields

$$
\Delta(x)=\frac{x^{2}\left(x^{10}+2 x^{9}+2 x^{8}+x^{7}-x^{5}+x^{3}+2 x^{2}+2 x+1\right)}{[2,3,5,7]\left(x^{2}+1\right)}>0 \quad \text { on } \quad(0,1],
$$

which proves our assertion. Let us pass to hyperbolic Coxeter quadrilaterals $P$ by noticing that they may have at most three right angles (cf. (2.1)). Hence, by the Lemma, we get the estimate

$$
H(x) \geq \frac{3}{[2]}+\frac{[2]}{[3]} .
$$

Therefore, (3.17), (3.19) and (3.6) imply that $(3,7)$ has minimal growth rate among all compact planar Coxeter triangles and quadrilaterals. Finally, let us look at compact Coxeter polygons with at least five vertices. Then, together with (3.17) and (3.6), we get the obvious estimate

$$
H(x) \geq \frac{5}{[2]}>H_{(3,7)}(x)
$$

which finishes the proof. 


\section{Growth rate versus covolume}

Consider a hyperbolic Coxeter group acting cocompactly on $\mathbb{H}^{2}$ or $\mathbb{H}^{3}$. Example 3 documents that the group $(3,7)$ has minimal growth rate and minimal covolume among all planar hyperbolic Coxeter groups. This observation leads to several further questions about connections between growth rates and covolumes in dimensions two and three. Some aspects will be discussed below.

4.1. The planar case. By restricting to the cocompact case, let us consider the question (cf. [GhHi, Problem 16]) whether there is a direct connection between the volumes of hyperbolic polygons and the asymptotic growth rates of the underlying Coxeter reflection groups. In general, the answer is NO according to the following propositions.

Proposition 1. There exist two infinite families $P_{k}$ and $Q_{k}, k \geq 4$, of compact hyperbolic Coxeter polygons such that $\tau_{P_{k}}=\tau_{Q_{k}}$ but $\left|\operatorname{vol}_{2}\left(P_{k}\right)-\operatorname{vol}_{2}\left(Q_{k}\right)\right| \rightarrow \infty$ for $k \rightarrow \infty$.

Proof. Let $k \geq 4$, and consider a hyperbolic $k$-gon $P_{k}$ all of whose angles equal $\pi / 3$, and a totally right-angled hyperbolic $(k+1)$-gon $Q_{k}$. By $(2.1)$ and by evaluating the total interior angle sum $\Omega$, one sees that the polygons $P_{k}$ and $Q_{k}$ exist for each $k \geq 4$. By (2.5) or (2.12), the corresponding growth functions can be computed and are equal to

$$
f_{P_{k}}(x)=\frac{[3]}{x^{2}-(k-1) x+1} \quad, \quad f_{Q_{k}}(x)=\frac{[2]^{2}}{x^{2}-(k-1) x+1} .
$$

Since the denominators in (4.1) are identical, the growth rates of $P_{k}$ and $Q_{k}$ coincide for each $k \geq 4$. By the defect formula for an $N$-gon, expressing the volume as $(N-2) \pi-\Omega$ (or by using (2.7)), one gets

$$
\operatorname{vol}_{2}\left(P_{k}\right)=\frac{2 \pi}{3}(k-3) \quad, \quad \operatorname{vol}_{2}\left(Q_{k}\right)=\frac{\pi}{2}(k-3) \quad,
$$

so that

$$
\left|\operatorname{vol}_{2}\left(P_{k}\right)-\operatorname{vol}_{2}\left(Q_{k}\right)\right|=\frac{\pi}{6}(k-3) \rightarrow \infty \quad \text { for } \quad k \rightarrow \infty \quad .
$$

Proposition 2. There exist two infinite families $R_{k}$ and $S_{k}, k \geq 2$, of compact hyperbolic Coxeter polygons such that $\operatorname{vol}_{2}\left(R_{k}\right)=\operatorname{vol}_{2}\left(S_{k}\right)$ but $\left|\tau_{R_{k}}-\tau_{S_{k}}\right| \rightarrow \infty$ for $k \rightarrow \infty$.

Proof. Let $R_{k}$ be a right-angled hyperbolic (4k)-gon, and let $S_{k}$ be a hyperbolic (3k)-gon with all angles equal to $\pi / 3$. Again, both families of polygons exist for $k \geq 2$. By the defect formula, one derives $\operatorname{vol}_{2}\left(R_{k}\right)=2 \pi(k-1)=\operatorname{vol}_{2}\left(S_{k}\right)$. For their growth functions, one obtains the expressions

$$
f_{R_{k}}(x)=\frac{[2,2]}{x^{2}-(4 k-2) x+1} \quad, \quad f_{S_{k}}(x)=\frac{[3]}{x^{2}-(3 k-1) x+1} .
$$


A straightforward computation for the roots of the denominator polynomials shows that $\left|\tau_{R_{k}}-\tau_{S_{k}}\right|$ grows like $k$ for $k \rightarrow \infty$. Acutally, one can even deduce that $\tau_{R_{k}} / \tau_{S_{k}} \rightarrow 4 / 3$ which shows that $\tau_{R_{k}}$ and $\tau_{S_{k}}$ are not asymptotically equivalent.

4.2. The spatial case. In contrast to the planar case, the group $(3,5,3)$ has minimal growth rate $\tau_{(3,5,3)} \simeq 1.35098$ but not minimal covolume among all Coxeter groups acting cocompactly on $\mathbb{H}^{3}$. In fact, consider the group $(4,3,5)$ with growth rate $\tau_{(4,3,5)} \simeq 1.35999$ which describes a compact Coxeter orthoscheme of volume slightly smaller than 0.03589 (cf. [Ke, Appendix]). Therefore, the group $(3,5,3)$ which is of volume slightly bigger than 0.03900 (cf. [Ke, Appendix]), cannot be a minimal volume compact hyperbolic Coxeter group or, more generally, a minimal volume compact hyperbolic orbifold of three dimensions. Recently, it has been proven by Gehring, Marshall and Martin (see [GM] and [MaM]) that the orbit space $Q$ of $\mathbb{H}^{3}$ by the $\mathbb{Z}_{2}$-extension of $(3,5,3)$ with volume $\operatorname{vol}_{3}(Q) \simeq 0.01953$ is the compact hyperbolic 3-orbifold of minimal volume.

As in (4.1), we can illustrate this discrepancy as follows. Consider the $k$-th Löbell polyhedron $L(k), k \geq 5$, all of whose dihedral angles are right (cf. [Ve]).

$k$-gonal face $F_{1}$

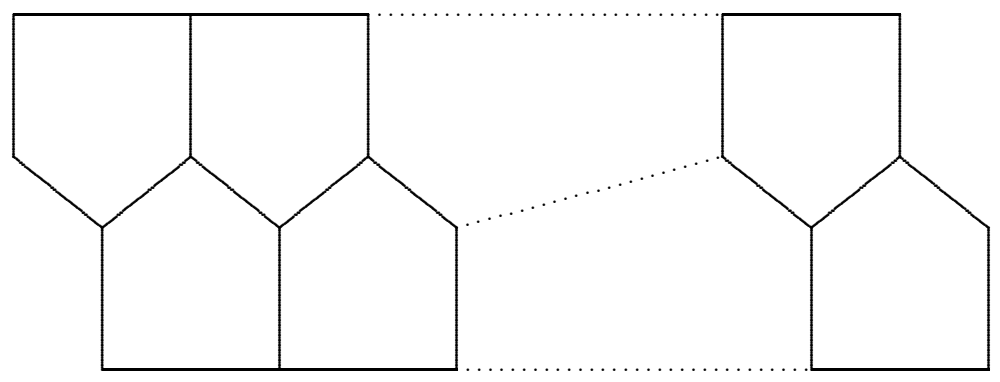

$k$-gonal face $F_{0}$

Figure 2. An unfolding of the Löbell polyhedron $L(k), k \geq 5$. The left-hand side is glued to the right-hand side.

Observe that the polyhedron $L(5)$ coincides with a totally right-angled hyperbolic dodecahedron. From the construction it follows that $L(k)$ has two $k$-gonal faces which are at the bottom and the top of two polygonal ribbons consisting of $k$ lateral pentagonal faces, each (see Figure 2). The polyhedron $L(k)$ has therefore $f_{2}=2 k+2$ faces, $f_{1}=6 k$ edges and $f_{0}=4 k$ vertices. By $[\mathrm{Ve},(7)]$, the volume of $L(k)$ behaves asymptotically according to

$$
\operatorname{vol}_{3}(L(k)) \sim 10 k \nu_{3}
$$

where $\nu_{3} \simeq 1.01494$ is the volume of an ideal hyperbolic regular tetrahedron.

In the sequel, we shall construct infinite families of compact Coxeter polyhedra in $\mathbb{H}^{3}$, by glueing isometric copies of Löbell polyhedra along lateral pentagonal faces and along 
$k$-gonal (top and/or bottom) faces, and prove statements similar to Proposition 1 and Proposition 2.

Proposition 3. There exist two infinite families $G_{k}$ and $H_{k}, k \geq 5$, of compact hyperbolic Coxeter polyhedra such that $\tau_{G_{k}}=\tau_{H_{k}}$ but $\left|\operatorname{vol}_{3}\left(G_{k}\right)-\operatorname{vol}_{3}\left(H_{k}\right)\right| \rightarrow \infty$ for $k \rightarrow \infty$.

Proof. Let $k \geq 5$, and denote by $G_{k}:=L(2 k)$ the $(2 k)$-th Löbell polyhedron $L(2 k)$ with $f$-vector $\left(f_{0}, f_{1}, f_{2}\right)=(8 k, 12 k, 4 k+2)$. Next, take a Löbell polyhedron $L(k)$ and glue two further, isometric copies of it to its $k$-gonal bottom and top faces $F_{0}$ and $F_{1}$. Since $L(k)$ is right-angled, the resulting polyhedron $H_{k}$ exists, is right-angled and has $f$-vector $(8 k, 12 k, 4 k+2)$, which is identical to the one of $G_{k}$. In fact, the glueing process makes the edges, together with their vertices, of the glued faces $F_{0}$ and $F_{1}$ disappear.

Now, both Coxeter polyhedra $G_{k}$ and $H_{k}$ are compact and right-angled so that their associated growth functions can be computed according to the formula (see [D, Example 17.4.3])

$$
f(x)=\frac{[2]^{3}}{1-\left(f_{2}-3\right) x+\left(f_{2}-3\right) x^{2}-x^{3}} .
$$

Therefore, we obtain

$$
f_{G_{k}}(x)=\frac{[2]^{3}}{1-(4 k-1) x+(4 k-1) x^{2}-x^{3}}=f_{H_{k}}(x)
$$

which implies that $\tau_{G_{k}}=\tau_{H_{k}}$. By construction, we have the volume identity $\operatorname{vol}_{3}\left(H_{k}\right)=$ $3 \operatorname{vol}_{3}\left(L_{k}\right)$ so that formula (4.3) leads to the asymptotic laws

$$
\operatorname{vol}_{3}\left(G_{k}\right) \sim 20 k \nu_{3} \quad, \quad \operatorname{vol}_{3}\left(H_{k}\right) \sim 30 k \nu_{3} \quad
$$

for large $k$. This finishes the proof.

Proposition 4. There exist two infinite families $L_{k}$ and $M_{k}, k \geq 6$, of compact hyperbolic Coxeter polyhedra such that $\operatorname{vol}_{3}\left(L_{k}\right)=\operatorname{vol}_{3}\left(M_{k}\right)$ but $\left|\tau_{L_{k}}-\tau_{M_{k}}\right| \rightarrow \infty$ for $k \rightarrow \infty$.

Proof. Let $k \geq 6$, and consider the double $L_{k}$ of $L(k)$ which arises by glueing two isometric copies of $L(k)$ along one $k$-gonal face. Then, $\operatorname{vol}_{3}\left(L_{k}\right)=2 \operatorname{vol}_{3}(L(k))$. In a similar way, glue two copies of $L(k)$ along one (right-angled) pentagonal face and call the new compact right-angled polyhedron $M_{k}$. It follows that $\operatorname{vol}_{3}\left(M_{k}\right)=2 \operatorname{vol}_{3}(L(k))=\operatorname{vol}_{3}\left(L_{k}\right)$.

Furthermore, it is not difficult to identify the number of faces of $L_{k}$ and $M_{k}$ with $f_{2}\left(L_{k}\right)=$ $3 k+2$ and $f_{2}\left(M_{k}\right)=4 k-3$. By $(4.4)$, this leads to the growth functions

$$
\begin{aligned}
f_{L_{k}}(x) & =\frac{[2]^{3}}{1-(3 k-1) x+(3 k-1) x^{2}-x^{3}} \\
f_{M_{k}}(x) & =\frac{[2]^{3}}{1-(4 k-6) x+(4 k-6) x^{2}-x^{3}}
\end{aligned} .
$$


The computation of the roots (see [R, Discriminants]) for the denominator polynomials in (4.6) yields

$$
\begin{aligned}
\tau_{L_{k}} & =\frac{1}{2}\left(3 k-2+\sqrt{9 k^{2}-12 k}\right), \\
\tau_{M_{k}} & =\frac{1}{2}\left(4 k-7+\sqrt{16 k^{2}-56 k+45}\right) .
\end{aligned}
$$

As a consequence, $\left|\tau_{L_{k}}-\tau_{M_{k}}\right| \rightarrow \infty$ for $k \rightarrow \infty$. More precisely, one deduces from (4.7) that

$$
\frac{\tau_{L_{k}}}{\tau_{M_{k}}} \longrightarrow \frac{3}{4} \quad \text { for } \quad k \rightarrow \infty
$$

which shows that the quantities $\tau_{L_{k}}$ and $\tau_{M_{k}}$ are not asymptotically equivalent.

\section{Bibliography}

[Ca] J. W. Cannon, The growth of the closed surface groups and the compact hyperbolic Coxeter groups, preprint.

[CaW] J. W. Cannon, P. Wagreich, Growth functions of surface groups, Math. Ann. 293 (1992), $239-257$.

[CD] R. Charney, M. W. Davis, Reciprocity of growth functions of Coxeter groups, Geom. Dedicata 39 (1991), 373-378.

[ChFr] T. Chinburg, E. Friedman, The smallest arithmetic hyperbolic three-orbifold, Invent. Math. 86 (1986), 507-527.

[Co] H. S. M. Coxeter, Discrete groups generated by reflections, Ann. Math. 35 (1934), 588-621.

[CoMo] H. S. M. Coxeter, W. O. J. Moser, Generators and relations for discrete groups, Springer-Verlag, 1980.

[D] M. W. Davis, The geometry and topology of Coxeter groups, London Mathematical Society Monographs, Princeton University Press, 2008.

[FlPl] W. J. Floyd, S. P. Plotnick, Symmetries of planar growth functions of Coxeter groups. Invent. Math. 93 (1988), 501-543.

[GM] F. W. Gehring, G. J. Martin, Minimal co-volume hyperbolic lattices I: The spherical points of a Kleinian group, Ann. Math. 170 (2009), 123-161.

[GhHi] E. Ghate, E. Hironaka, The arithmetic and geometry of Salem numbers, Bull. Amer. Math. Soc. 38 (2001), 293-314. 
[He] G. J. Heckman, The volume of hyperbolic Coxeter polytopes of even dimension, Indag. Math. (N.S.) 6 (1995), 189-196.

[Hi] E. Hironaka, The Lehmer polynomial and pretzel knots, Can. Math. Soc. Bulletin 44 (2001), 440-451.

[Im] H.-C. Im Hof, Napier cycles and hyperbolic Coxeter groups, Bull. Soc. Math. Belg. Sér. A 42 (1990), 523-545.

[In] T. Inoue, Organizing volumes of right-angled hyperbolic polyhedra, Algebr. Geom. Topol. 8 (2008), 1523-1565.

[Ka] I. M. Kaplinskaja, Discrete groups generated by reflections in the faces of simplicial prisms in Lobachevsii spaces, Math. Notes 15 (1974), 88-91.

[Ke] R. Kellerhals, On the volume of hyperbolic polyhedra Math. Ann. 285 (1989), 541-569.

[KeP] R. Kellerhals, G. Perren, On the growth of cocompact hyperbolic Coxeter groups, European Journal of Combinatorics 32 (2011), 1299-1316.

[MaM] T. H. Marshall, G. J. Martin, Minimal co-volume hyperbolic lattices, II: Simple torsion in a Kleinian group, to appear in Ann. Math. 2012.

[Mi] J. Milnor, A note on curvature and fundamental group, J. Diff. Geom. 2 (1968), 1-7.

[Pa] W. Parry, Growth series of Coxeter groups and Salem numbers, J. Algebra 154 (1993), 406-415.

[Pr] V. V. Prasolov, Polynomials, Springer-Verlag, 2004.

[R] J. Rotman, Galois Theory, Springer-Verlag, 1998.

[Si] C. L. Siegel, Some remarks on discontinuous groups, Ann. Math. 46 (1945), 708-718.

[So] L. Solomon, The orders of the finite Chevalley groups, J. Algebra 3 (1966), 376-393.

[St] R. Steinberg, Endomorphisms of linear algebraic groups, Mem. Amer. Math. Soc. 80 (1968).

[Ve] A. Y. Vesnin, Volumes of hyperbolic Löbell 3-manifolds, Math. Notes 64 (1998), 15-19.

[Vi1] E. B. Vinberg, The absence of crystallographic groups of reflections in Lobachevsky spaces of large dimensions, Trans. Moscow Math. Soc. 47 (1985), 75-112.

[Vi2] E. B. Vinberg, Hyperbolic reflection groups, Russian Math. Surveys 40 (1985), 31-75.

[W] R. L. Worthington, The growth series of compact hyperbolic Coxeter groups with 4 and 5 generators, Canad. Math. Bull. 41 (1998), 231-239. 